\title{
Weak spectral synthesis in Fourier algebras of coset spaces
}

by

\author{
Eberhard KANIUth (Paderborn)
}

\begin{abstract}
Let $G$ be a locally compact group, $K$ a compact subgroup of $G$ and $A(G / K)$ the Fourier algebra of the coset space $G / K$. Applying results from [E. Kaniuth, Weak spectral synthesis in commutative Banach algebras, J. Funct. Anal. 254 (2008), 9871002], we establish injection and localization theorems relating weak spectral sets and weak Ditkin sets for $A(G / K)$ to such sets for $A(H / H \cap K)$, where $H$ is a closed subgroup of $G$. We also prove some results towards the analogue of Malliavin's theorem for weak spectral synthesis in $A(G / K)$ and give illustrating examples.
\end{abstract}

Introduction. Let $A$ be a regular and semisimple commutative Banach algebra with structure space $\Delta(A)$ and Gelfand transform $a \mapsto \widehat{a}$. For any subset $M$ of $A$, the hull $h(M)$ of $M$ is defined by $h(M)=\{\varphi \in \Delta(A)$ : $\varphi(M)=\{0\}\}$. Associated to each closed subset $E$ of $\Delta(A)$ are two distinguished ideals with hull equal to $E$, namely

$$
k(E)=\{a \in A: \widehat{a}(\varphi)=0 \text { for all } \varphi \in E\},
$$

the kernel of $E$, and

$$
j(E)=\{a \in A: \widehat{a} \text { has compact support disjoint from } E\} .
$$

Then $k(E)$ is the largest ideal with hull $E$, and $j(E)$ is the smallest such ideal. Recall that $E$ is a spectral set (or set of synthesis) if $k(E)=\overline{j(E)}$ (equivalently, $k(E)$ is the only closed ideal with hull equal to $E$ ). One says that spectral synthesis holds for $A$ if every closed subset of $\Delta(A)$ is a spectral set. Moreover, $E$ is a Ditkin set if $a \in \overline{a j(E)}$ for every $a \in k(E)$.

A weaker form of spectral sets and spectral synthesis was introduced and studied in [26], motivation arising from the union problem for sets of synthesis. A closed subset $E$ of $\Delta(A)$ is called a weak spectral set if there exists $n \in \mathbb{N}$ such that $a^{n} \in \overline{j(E)}$ for every $a \in k(E)$. The smallest such number $n$ is denoted $\xi(E)$. When this happens for each $E$, we say that weak

2010 Mathematics Subject Classification: Primary 43A45; Secondary 43A30, 46J10, 46J20. Key words and phrases: locally compact group, coset space, Fourier algebra, weak spectral set, weak Ditkin set, weak spectral synthesis, injection theorem, localization theorem. 
spectral synthesis holds for $A$. The importance of these latter notions is due to the fact that the class of weak spectral sets is usually considerably larger than the class of spectral sets and that there are many commutative Banach algebras for which weak synthesis holds, whereas synthesis fails. Notably, for $n \in \mathbb{N}$, the unit sphere $S^{n-1}$ in $\mathbb{R}^{n}=\Delta\left(L^{1}\left(\mathbb{R}^{n}\right)\right)$ satisfies $\xi\left(S^{n-1}\right)=$ $\lfloor(n+1) / 2\rfloor([24)$. For further examples see [8, Section 1]. Generalizing the notion of a Ditkin set, we call $E$ a weak Ditkin set if there exists $n \in \mathbb{N}$ such that $a^{n} \in \overline{a^{n} j(E)}$ for each $a \in k(E)$, and $\eta(E)$ will stand for the minimal such $n$. Weak spectral synthesis was also studied in [18] and, more recently, in [7], 8] and [16].

Let $G$ be a locally compact group and $K$ a compact subgroup of $G$. The Fourier algebra $A(G / K)$ of the coset space $G / K$ was first systematically studied by Forrest [3]. $A(G / K)$ is a semisimple and regular commutative Banach algebra whose spectrum can be canonically identified with $G / K$. There is a substantial body of contributions, of which we only mention [1, [3], [10, [11], [12, 15], [17] and [23], to spectral synthesis in Fourier algebras $A(G)$. Recently, the algebras $A(G / K)$ have gained considerable attention and have been investigated in several aspects, such as spectral synthesis problems and various kinds of amenability (see [4], [5], [16], [17]).

In [8] we have established injection theorems for weak spectral sets and weak Ditkin sets for general semisimple and regular commutative Banach algebras $A$ and quotient algebras $A / I$. These theorems are applied in Section 2 to Fourier algebras $A(G / K)$ and quotient algebras $A(H / H \cap K)$ where $H$ is a closed subgroup of $G$. Our results (Theorem 2.2 ) are partly even new when $K$ is trivial, that is, for ordinary Fourier algebras. In Section 3 we apply Theorem 2.2 and further results from [8] to deduce a characterization of weak spectral sets and weak Ditkin sets for $A(G / K)$ in terms of such sets for $A(H / H \cap K)$, where $H$ is an open subgroup of $G$.

It was shown in [8] that weak spectral synthesis holds for the Fourier algebra $A(G)$ of any locally compact group $G$ if and only if $G$ is discrete. It is tempting to conjecture that weak spectral synthesis holds for $A(G / K)$ if and only if $K$ is open in $G$. However, this appears to be a very difficult problem and we obtain only partial results towards this conjecture (Theorem 4.3). The preceding problem is closely related to the question of how large a subalgebra of $A(H / H \cap K)$ the restriction algebra $\left.A(G / K)\right|_{H}$ is. In the last section we address this question in the special case of a semidirect product $G=H \rtimes K$.

1. Preliminaries. Let $G$ be a locally compact group. Eymard [2] has introduced and intensively studied the Fourier algebra $A(G)$ and the FourierStieltjes algebra $B(G)$ of $G$. The algebra $B(G)$ is the collection of all coefficient functions of continuous unitary representations of $G$ and can be 
identified with the dual space of the group $C^{*}$-algebra $C^{*}(G)$. Equipped with pointwise operations and the norm defined by this duality, $B(G)$ is a Banach algebra. The Fourier algebra $A(G)$ is the closed ideal of $B(G)$ generated by all functions in $B(G)$ with compact support. If $G$ is abelian with dual group $\widehat{G}$, then $B(G)$ is isometrically isomorphic to the measure algebra $M(\widehat{G})$ via the Fourier-Stieltjes transform, and this isomorphism maps $A(G)$ onto the $L^{1}$-algebra $L^{1}(\widehat{G})$. As shown in [2], $A(G)$ is a semisimple and regular commutative Banach algebra whose spectrum $\Delta(A(G))$ can be identified with $G$ through assigning to $x \in G$ the homomorphism $u \mapsto u(x)$, $u \in A(G)$. Recall that $A(G)$ has a bounded approximate identity if and only if $G$ is amenable (see [19, Theorem 10.4]).

Let $K$ be a compact subgroup of the locally compact group $G$ and let $G / K$ be the space of left cosets of $K$ in $G$, equipped with the quotient topology. The Fourier algebra $A(G / K)$ of $G / K$ was extensively studied in [3]. $A(G / K)$ can be simultaneously viewed as an algebra of continuous functions on $G / K$ and as a closed subalgebra of $A(G)$. We always take the latter view. Then

$$
A(G / K)=\{u \in A(G): u(x k)=u(x) \text { for all } x \in G, k \in K\} .
$$

In 22] it was shown that the spaces $A(G / K)$ are precisely the norm closed left translation invariant $*$-subalgebras of $A(G)$. Suppose that Haar measure $|\cdot|$ of $K$ is normalized so that $|K|=1$. Then there is a canonical projection $p_{K}$ from $A(G)$ onto $A(G / K)$ defined by

$$
p_{K}(u)(x K)=\int_{K} u(x k) d k, \quad x \in G, u \in A(G) .
$$

Thus $p_{K}(u)$ is nothing but the Bochner integral $\int_{K} R_{k}(u) d k \in A(G)$, where $R_{k}(u)(x)=u(x k)$

Theorem 1.1 ([3, Theorems 4.1 and 4.2]). $A(G / K)$ is a regular and semisimple commutative Banach algebra with $\Delta(A(G / K))=G / K$. More precisely, the map $x K \mapsto \varphi_{x K}$, where $\varphi_{x K}(u)=u(x K)$ for $u \in A(G / K)$, is a homeomorphism from $G / K$ onto $\Delta(A(G / K))$. Furthermore, $A(G / K)$ has a bounded approximate identity if and only if $G$ is amenable.

In addition to Theorem 1.1, we shall need a series of lemmas. The first of these is a simple consequence of [3, Proposition 3.10].

Lemma 1.2. Let $H$ and $K$ be closed subgroups of $G$ with $K$ compact. If $K \subseteq H$, then $A(H / K)$ is isometrically isomorphic to $A(G / K) / k(H K)$.

Proof. The map $r: A(G / K) \rightarrow A(H),\left.u \mapsto u\right|_{H}$, has kernel $k(H K)$, is norm decreasing and $r(A(G / K)) \subseteq A(H / H \cap K)=A(H / K)$. Given $v \in A(H)$, by Proposition 3.10 of [3] there exists an extension $u \in A(G / K)$ 
of $v$ with $\|u\|_{A(G)}=\|v\|_{A(H)}$. So $r$ induces an isometric isomorphism from $A(G / K) / k(H K)$ onto $A(H / K)$.

Of course, the question arises of when, more generally, for a closed subgroup $H$ of $G$ and a compact subgroup $K$ of $G$, the restriction map $\left.u \mapsto u\right|_{H}$ from $A(G / K)$ into $A(H / H \cap K)$ is surjective. We shall present two such cases in Lemma 2.3. For the reader who is less familiar with spectral synthesis questions, we mention [9], 20] and [21] as references.

Lemma 1.3 ([2, Proposition 4.3]). Let $K$ be a compact subgroup of $G$, $q: G \rightarrow G / K$ the quotient map and $E$ a closed subset of $G / K$. If $q^{-1}(E)$ is a (weak) spectral set for $A(G)$, then $E$ is a (weak) spectral set for $A(G / K)$.

Lemma 1.4. Let $K$ and $C$ be compact subgroups of $G$ and assume that $C$ is normal in $G$. Let $q: G / K \rightarrow G / K C$ denote the quotient map. Then $\xi(E) \leq \xi\left(q^{-1}(E)\right)$ for every closed subset $E$ of $G / K C$. In particular, if weak spectral synthesis (respectively, spectral synthesis) holds for $A(G / K)$, then it also holds for $A((G / C) /(K C / C))$.

Proof. We can assume $n=\xi\left(q^{-1}(E)\right)<\infty$. Let $u \in A((G / C) /(K C / C))$ be such that $\left.u\right|_{E}=0$. Then $\left.(u \circ q)\right|_{q^{-1}(E)}=0$ and

$$
u \circ q(x k)=u(x k C)=u(x C)=u \circ q(x)
$$

for all $x \in G$ and $k \in K$. Given $\epsilon>0$, by hypothesis there exists $v \in A(G / K)$ such that $\left\|(u \circ q)^{n}-v\right\|_{A(G / K)}<\epsilon$ and $v$ vanishes on some open set $U$ with $q^{-1}(E) \subseteq U$ and $(G / K) \backslash U$ compact. Since $q^{-1}(E)=q^{-1}(E) C$ and $C$ is compact, a simple topological argument shows that we can assume that $U=U C \subseteq G / K$. Define $w \in A(G / C)$ by

$$
w(x C)=\int_{C} v(x c) d c, \quad x \in G .
$$

Then $w$ vanishes on the open set $q(U), E \subseteq q(U)$, and $(G / K C) \backslash q(U)$ is compact. Moreover, for $x \in G, k \in K$ and $c \in C$,

$$
\begin{aligned}
w(x k c C) & =\int_{C} v\left(x k c c^{\prime}\right) d c^{\prime}=\int_{C} v\left(x k c^{\prime} k^{-1} k\right) d c^{\prime} \\
& =\int_{C} v\left(x k c^{\prime} k^{-1}\right) d c^{\prime}=\int_{C} v\left(x c^{\prime}\right) d c^{\prime}=w(x C),
\end{aligned}
$$

so that $w \in A((G / C) /(K C / C))$. Finally,

$$
\begin{aligned}
\left\|u^{n}-w\right\|_{A(G / C)} & =\left\|u^{n} \circ q-w \circ q\right\|_{A(G)}=\left\|(u \circ q)^{n}-w \circ q\right\|_{A(G)} \\
& =\left\|\int_{C} R_{C}(u \circ q)^{n} d c-\int_{C} R_{c} v d c\right\|_{A(G)} \\
& \leq \int_{C}\left\|R_{C}\left((u \circ q)^{n}-v\right)\right\|_{A(G)} d c=\left\|(u \circ q)^{n}-v\right\|_{A(G)} .
\end{aligned}
$$

So $w \in j(E)$ and $\left\|u^{n}-w\right\|_{A(G / C)}<\epsilon$. 
The following lemma is nothing but a slight extension of Proposition 4.3 of 3 ].

LEMMA 1.5. Let $K_{1}$ and $K_{2}$ be compact subgroups of $G$ such that $K_{1} \subseteq K_{2}$. If weak spectral synthesis holds for $A\left(G / K_{1}\right)$, then it also holds for $A\left(G / K_{2}\right)$.

Proof. Let $E$ be a closed subset of $G / K_{2}$ and let $q: G / K_{1} \rightarrow G / K_{2}$ denote the quotient map. If $u \in k(E) \subseteq A\left(G / K_{2}\right)$, then $u \circ q \in k(E \circ q) \subseteq$ $A\left(G / K_{1}\right)$, and hence there exist $m \in \mathbb{N}$ and a sequence $\left(v_{n}\right)_{n} \subseteq j\left(q^{-1}(E)\right)$ such that $\left\|(u \circ q)^{m}-v_{n}\right\|_{A(G)} \rightarrow 0$. Let $w_{n}=\int_{K_{2}} R_{k}\left(v_{n}\right) d k \in A\left(G / K_{2}\right)$, $n \in \mathbb{N}$. Then

$$
\left\|(u \circ q)^{m}-w_{n}\right\|_{A(G)} \leq\left\|(u \circ q)^{m}-v_{n}\right\|_{A(G)} \rightarrow 0,
$$

where Haar measure of $K_{2}$ is normalized. Moreover, $w_{n} \in j\left(q^{-1}(E)\right)$ since supp $w_{n} \subseteq q^{-1}\left(q\left(\operatorname{supp} v_{n}\right)\right)$, which is a compact set, and since

$$
q^{-1}\left(q\left(\operatorname{supp} v_{n}\right)\right) \cap q^{-1}(E) \neq \emptyset
$$

implies that supp $v_{n} \cap q^{-1}(E) \neq \emptyset$, which is impossible.

2. Injection theorems. Let $G$ be a locally compact abelian group and $H$ a closed subgroup of $G$. Then $L^{1}(G / H)$ is a quotient of $L^{1}(G)$ and $\widehat{G / H}=\Delta\left(L^{1}(G / H)\right)$ embeds canonically into $\widehat{G}=\Delta\left(L^{1}(G)\right)$. Then a closed subset of $\widehat{G / H}$ is a spectral set (respectively, Ditkin set) for $L^{1}(G / H)$ if and only if it is a spectral set (respectively, Ditkin set) for $L^{1}(G)$ (see [20, Theorems 7.3.15 and 7.4.13]). For obvious reasons, such results are referred to as injection theorems. The same problem naturally arises in the general context of a regular and semisimple commutative Banach algebra $A$ and a closed ideal $I$ of $A$, and it is worthwhile to consider weak spectral sets and weak Ditkin sets rather than just spectral sets and Ditkin sets. However, as the reader might expect, some additional hypotheses, which are automatically satisfied in the group algebra situation, have to be placed on $A$ and $I$. Such injection theorems have been established recently in [8]. Below, we apply these results of [8] to obtain injection theorems for the Fourier algebras $A(G / K)$ of coset spaces $G / K$ and quotient algebras of the form $A(H / H \cap K)$, where $H$ is a closed subgroup of $G$.

Lemma 2.1. Let $G$ be an amenable locally compact group, $H$ a closed subgroup of $G$, and $K$ a compact subgroup of $G$. Suppose that $H K$ is a subgroup of $G$. Given $u \in A(G / K)$ and $\epsilon>0$, there exists $v \in A(G / K)$ such that $v$ vanishes in a neighbourhood of $H K$ and

$$
\|u-v u\|_{A(G)}<3\left\|\left.u\right|_{H K}\right\|_{A(H K)}+\epsilon .
$$

In particular, the ideal $k(H K / K)=\left\{u \in A(G / K):\left.u\right|_{H}=0\right\}$ of $A(G / K)$ has an approximate identity with norm bound 2 consisting of functions which vanish in a neighbourhood of $H K$. 
Proof. Suppose that we have found $v \in A(G)$ with the specified properties. Then $p_{K}(v) \in A(G / K)$ also vanishes in a neighbourhood of $H K$ and, since $u=p_{K}(u)$,

$$
\left\|u-p_{K}(v) u\right\|_{A(G)}=\left\|\int_{K}\left(R_{k}(u)-R_{k}(v u)\right) d k\right\|_{A(G)} \leq\|u-v u\|_{A(G)} .
$$

Since, by [3, Lemma 3.8],

$$
\left\|\left.u\right|_{H K}\right\|_{A(H K)}=\inf \left\{\|u+w\|_{A(G)}: w \in k(H K)\right\},
$$

we find $w \in k(H K)$ such that

$$
\|u-w\|_{A(G)}<\left\|\left.u\right|_{H K}\right\|_{A(H K)}+\epsilon / 4 .
$$

Now, $H K$ is a set of synthesis [23, Theorem 3] (see also [6]) and, since $G$ is amenable, the ideal $k(H K)$ of $A(G)$ has a bounded approximate identity of norm bound 2 (see [4, Theorem 2.6]). Hence there exists $v \in j(H K)$ with $\|v\|_{A(G)} \leq 2$ and $\|w-v w\|_{A(G)}<\epsilon / 4$. It follows that

$$
\begin{aligned}
\|u-u v\|_{A(G)} & \leq\|u-w\|_{A(G)}+\|w-v w\|_{A(G)}+\|v(w-u)\|_{A(G)} \\
& <\left\|\left.u\right|_{H K}\right\|_{A(H K)}+\frac{\epsilon}{4}+\frac{\epsilon}{4}+2\left(\left\|\left.u\right|_{H K}\right\|_{A(H K)}+\frac{\epsilon}{4}\right) \\
& =3\left\|\left.u\right|_{H K}\right\|_{A(H K)}+\epsilon,
\end{aligned}
$$

as was to be shown. The final statement of the lemma is now clear.

Most likely, the number 3 in the preceding lemma is not the best possible bound. This is suggested by the fact that 3 can be replaced by 1 when $G$ is abelian [20, Lemma 7.4.14]. We can now state the injection theorem.

THEOREM 2.2. Let $G$ be a locally compact group, $H$ be a closed subgroup of $G$, and $K$ be a compact subgroup of $G$. Suppose that the map $\left.u \mapsto u\right|_{H}$ from $A(G / K)$ into $A(H / H \cap K)$ is surjective. Let $E$ be a closed subset of $H / H \cap K$ and let $i: H / H \cap K \rightarrow G / K$ denote the inclusion map $h(H \cap K) \mapsto h K$, $h \in H$.

(i) If $i(E)$ is a weak spectral set (respectively, weak Ditkin set) for $A(G / K)$, then $E$ is is a weak spectral set (respectively, weak Ditkin set) for $A(H / H \cap K)$. Moreover,

$$
\xi(E) \leq \xi(i(E)) \quad \text { (respectively, } \eta(E) \leq \eta(i(E))) .
$$

(ii) Suppose that $H K / K$ is a set of synthesis for $A(G / K)$. If $E$ is a weak spectral set for $A(H / H \cap K)$, then $i(E)$ is a weak spectral set for $A(G / K)$ and $\xi(i(E)) \leq \xi(E)$. In particular, this conclusion holds if $H K$ is a subgroup of $G$.

(iii) Suppose that $G$ is amenable and $H K$ is a subgroup of $G$. If $E$ is a weak Ditkin set for $A(H / H \cap K)$, then $i(E)$ is a weak Ditkin set for $A(G / K)$ and $\eta(i(E)) \leq \eta(E)^{2}$. 
Proof. Since $r:\left.u \mapsto u\right|_{H}$ is a continuous homomorphism of $A(G / K)$ onto $A(H / H \cap K)$ and the kernel of $r$ equals $J=A(G / K) \cap k(H K)$, by the open mapping theorem $A(H / H \cap K)$ is topologically isomorphic to $A(G / K) / J$.

Therefore, the statements in (i) follow from Theorem 2.2(i) and Theorem 2.5 (i) of [8], respectively.

(ii) The first statement is a consequence of [8, Theorem 2.2(ii)]. If $H K$ is a subgroup of $G$, then it is a set of synthesis for $A(G)$ and hence $H K / K$ is a set of synthesis for $A(G / K)$ by Lemma 1.3.

(iii) Since $G$ is amenable and $H K$ is a subgroup of $G$, Lemma 2.1 shows that $A=A(G / K)$ and the ideal $I=k(H K / K)$ satisfy the hypotheses of Theorem 2.5(ii) of [8] with $m=1$ and $C=3$. It follows that $i(E)$ is a weak Ditkin set for $A(G / K)$ and that $\eta(i(E)) \leq \eta(E)^{2}$.

When $K=\{e\}$, assertions (i) and (ii) of Theorem 2.2 were known before (compare [10, Theorem 3.5] and [11, Theorem 3.4]). Part (iii), however, is entirely new, even in the case $K=\{e\}$.

In Theorem 2.2(ii), the hypothesis that $H K / K$ be a set of synthesis could be weakened to the effect that it be a weak spectral set, with the conclusion that $\xi(i(E)) \leq \xi(E) \xi(H K / K)$ (see [8, Theorem 2.2]).

If $K \subseteq H$, then the map $\left.u \mapsto u\right|_{H}$ from $A(G / K)$ into $A(H / H \cap K)$ is surjective (Lemma 1.2). We now discuss two more cases in which this is true.

Lemma 2.3. Let $H$ be a closed subgroup and $K$ a compact subgroup of $G$. The restriction map $r: A(G / K) \rightarrow A(H / H \cap K),\left.u \mapsto u\right|_{H}$, is surjective if either $H$ is contained in the normalizer of $K$ in $G$ or $H$ is open in $G$.

Proof. Suppose first that $H$ is contained in the normalizer of $K$ in $G$. Then $H K$ is a closed subgroup of $G, K$ is normal in $H K$ and the map

$$
\phi: H / H \cap K \rightarrow H K / K, \quad h(H \cap K) \mapsto h K,
$$

is a continuous bijective homomorphism. Since $K$ is compact and $A(H) \subseteq$ $C_{0}(H)$, given $v \in A(H / H \cap K)$, there exists an open, $\sigma$-compact subgroup $L$ of $H$ such that $H \cap K \subseteq L$ and $v=0$ on $H \backslash L$. Then $L \cap K=H \cap K$ and $\left.v\right|_{L} \in A(L / L \cap K)$, and $\left.\phi\right|_{L / L \cap K}$ is a topological isomorphism of $L / L \cap K$ onto $L K / K$. Let $w$ denote the trivial extension of $v \circ \phi^{-1} \in A(L K / K)$ to all of $H K$. Then $w \in A(H K / K)$ and

$$
v=\widetilde{\left.v\right|_{L}}=\left(\widetilde{\left.v\right|_{L} \circ \phi^{-1}}\right) \circ \phi=w \circ \phi=\left.w\right|_{H} .
$$

There exists $w^{\prime} \in A(G)$ with $\left.w^{\prime}\right|_{H K}=w$. Then $u=p_{K}\left(w^{\prime}\right)$ belongs to $A(G / K)$ and $\left.u\right|_{H K}=w$ since $w \in A(H K / K)$ and $r(u)=v$.

Now assume that $H$ is open in $G$ and let $v \in A(H / H \cap K)$. Let Haar measure of $K$ be normalized and set

$$
u=\frac{1}{[K:(K \cap H)]} \int_{K} R_{k}(\widetilde{v}) d k \in A(G),
$$


where $\widetilde{v}$ denotes the trivial extension of $v$ to all of $G$, and $[K:(K \cap H)]$ is the index of $K \cap H$ in $K$. Then, since $|K \cap H|=[K:(K \cap H)]^{-1}$, for $h \in H$ we have

$$
u(h)=\frac{1}{[K:(K \cap H)]} \int_{K \cap H} v(h k) d k=v(h),
$$

so that $u$ extends $v$. Moreover, for arbitrary $x \in G$ and $a \in K$,

$$
\begin{aligned}
{[K:(K \cap H)] u(x a) } & =\int_{K} \widetilde{v}(x a k) d k=\int_{K \cap a^{-1} x^{-1} H} v(x a k) d k \\
& =\int_{K \cap x^{-1} H} v(x k) d k=\int_{K} \widetilde{v}(x k) d k=[K:(K \cap H)] u(x) .
\end{aligned}
$$

This shows that $u \in A(G / K)$, and we are done.

A further comment is in order concerning the hypothesis in Theorem 2.2 (ii) that $H K / K$ be a set of synthesis for $A(G / K)$. This hypothesis is not only satisfied if $H K$ is a subgroup of $G$, but also if $H$ is open in $G$ and, in addition, $\emptyset$ is a Ditkin set for $A(G)$. This can be seen as follows. Recall that if $A$ is any regular and semisimple commutative Banach algebra, then the union of two disjoint spectral sets in $\Delta(A)$ is again a spectral set provided that $\emptyset$ is a Ditkin set. In the present situation, because $H$ is open and $K$ is compact, $H K$ is a finite union of right cosets of $H$ and hence is a set of synthesis for $A(G)$. Now apply Lemma 1.3 again. We also remind the reader that $\emptyset$ is a Ditkin set if $A(G)$ has a (possibly unbounded) approximate identity and so in particular if $G$ is amenable.

We state the most important special case of Theorem 2.2 as a corollary.

Corollary 2.4. Let $G$ be a locally compact group and let $H$ be a closed subgroup of $G$, and $K$ a compact subgroup of $H$. Let $E$ be a closed subset of $H / K$ and let $i: H / K \rightarrow G / K$ denote the inclusion map.

(i) $E$ is a weak spectral set for $A(H / K)$ if and only if $i(E)$ is a weak spectral set for $A(G / K)$. More precisely, $\xi(E)=\xi(i(E))$.

(ii) Suppose that $G$ is amenable. Then $E$ is a weak Ditkin set for $A(H / K)$ if and only if $i(E)$ is a weak Ditkin set for $A(G / K)$, and in this case

$$
\eta(E) \leq \eta(i(E)) \leq \eta(E)^{2} .
$$

In particular, $E$ is a Ditkin set for $A(H / K)$ if and only if $i(E)$ is a Ditkin set for $A(G / K)$.

In [3, Corollary 4.5] it was shown that singletons in $G / K$ are sets of synthesis for $A(G / K)$, and if $G$ is amenable, then finite subsets of $G / K$ are sets of synthesis. These results of course follow from Theorem 2.2. Indeed, taking $H=K$ we find that $\{K\}$ is a set of synthesis and so is each singleton in $G / K$. Furthermore, if $G$ is amenable then singletons in $G / K$ are even Ditkin sets and hence so are finite subsets of $G / K$. Theorem 2.2 in particular 
applies if $H$ is contained in the centre of $G$ and $H \cap K=\{e\}$. We present one such example.

EXAMPLE 2.5. Let $\mathbb{H}_{3}$ be the 3-dimensional Heisenberg group, realized as $\mathbb{C} \times \mathbb{R}$ with multiplication

$$
\left(z_{1}, t_{1}\right)\left(z_{2}, t_{2}\right)=\left(z_{1}+z_{2}, t_{1}+t_{2}+\frac{1}{2} \operatorname{Im}\left(z_{1} \bar{z}_{2}\right)\right),
$$

$z_{1}, z_{2} \in \mathbb{C}, t_{1}, t_{2} \in \mathbb{R}$. Let the circle group $\mathbb{T}$ act on $\mathbb{H}_{3}$ by

$$
w \cdot(z, t)=(w z, t), \quad w \in \mathbb{T},(z, t) \in \mathbb{H}_{3},
$$

and form the semidirect product $G=\mathbb{H}_{3} \rtimes \mathbb{T}$. This solvable group is often referred to as the reduced oscillator group. Let $K=\mathbb{T}$ and $H=\mathbb{R} \times \mathbb{T}$, where $\mathbb{R}$ is identified with the subgroup $\{(0, t): t \in \mathbb{R}\}$ of $\mathbb{H}_{3}$. Then a closed subset $E$ of $\mathbb{R}$ is a weak spectral (respectively, Ditkin) set for $A(\mathbb{R})=A(H / \mathbb{T}$ ) if and only if $i(E) \subseteq G / \mathbb{T}=\mathbb{H}_{3}$ is a weak spectral (respectively, Ditkin) set for $A(G / \mathbb{T})$. Moreover, $\xi(i(E))=\xi(E)$ and $\eta(i(E))=\eta(E)$. Of course, instead of $\mathbb{H}_{3}$ we can equally well consider the higher-dimensional Heisenberg groups $\mathbb{H}_{2 n+1}$, realized as $\mathbb{C}^{n} \times \mathbb{R}$, and the corresponding analogue of the oscillator group, that is, the semidirect product $\mathbb{H}_{2 n+1} \rtimes \mathbb{T}^{n}$, where $\mathbb{T}^{n}$ acts on $\mathbb{C}^{n}$ componentwise.

3. A localization theorem. In this section we apply Theorem 2.2 and results from [8] to deduce some kind of localization theorem which characterizes weak spectral sets and weak Ditkin sets for $A(G / K)$ in terms of such sets for $A(H / H \cap K)$ when $H$ is open in $G$.

LEMMA 3.1. Let $H$ be an open subgroup of $G$, and $K$ a compact subgroup of $H$. If $E \subseteq G / K$ is a weak Ditkin set (respectively, weak spectral set) for $A(G / K)$, then $E \cap H / K$ is a weak Ditkin set (respectively, weak spectral set) for $A(H / K)$ and $\eta(E \cap H / K) \leq \eta(E)$ (respectively, $\xi(E \cap H / K) \leq \xi(E))$.

Proof. Let $E$ be a weak Ditkin set for $A(G / K)$ and $m=\eta(E)$, and let $u \in A(H / K)$ be such that $\left.u\right|_{E \cap H / K}=0$. Let $\widetilde{u}$ denote the trivial extension of $u$ to all of $G / K$. Then

$$
\widetilde{u} \in k((E \cap H / K) \cup(G / K \backslash H / K)) \subseteq k(E)
$$

and hence, given $\epsilon>0$, there exists $v \in j(E)$ such that $\left\|\widetilde{u}^{m}-\widetilde{u}^{m} v\right\|_{A(G / K)} \leq \epsilon$. It follows that $\left.v\right|_{H / K} \in j(E \cap H / K)$ and

$$
\left\|u^{m}-u^{m}\left(\left.v\right|_{H / K}\right)\right\|_{A(H / K)} \leq\left\|\widetilde{u}^{m}-\widetilde{u}^{m} v\right\|_{A(G / K)} \leq \epsilon .
$$

Since $\epsilon>0$ is arbitrary, this shows that $E \cap H / K$ is a weak Ditkin set for $A(H / K)$ with $\eta(E \cap H / K) \leq \eta(E)$.

The argument for weak spectral sets is similar, in fact, even simpler.

LEMMA 3.2. Let $G$ be an amenable locally compact group and let $H$ be an open subgroup of $G$, and $K$ a compact subgroup of $H$. Let $E$ be a closed 
subset of $G / K$ and suppose that for every $a \in G$, the set $a E \cap H / K$ is a weak Ditkin set for $A(H / K)$ and that $\eta(a E \cap H / K) \leq N$ for some $N \in \mathbb{N}$ and all $a \in G$. Then $E$ is a weak Ditkin set for $A(G / K)$ and $\eta(E) \leq N^{2}$.

Proof. Let $u \in k(E)$. Since $u \in C_{0}(G), u$ is concentrated on countably many cosets of $H$. So we find a $\sigma$-compact open subgroup $L$ of $G$ such that $H \subseteq L$ and $\left.u\right|_{G \backslash L}=0$. Let $A \subseteq G$ be a representative system for the left cosets of $H$ in $L$, and for $a \in A$, let $E_{a}=a E \cap H / K$. By hypothesis, each $E_{a}$ is a weak Ditkin set for $A(H / K)$ and $\eta\left(E_{a}\right) \leq N$. Let $i$ denote the inclusion map of $L / K$ into $G / K$. Since $L$ is amenable, Theorem 2.2(ii) applies and shows that $i\left(E_{a}\right)$ is a weak Ditkin set for $A(L / K)$ and $\eta\left(i\left(E_{a}\right)\right) \leq N^{2}$.

Now if $F \subseteq L / K$ is a weak Ditkin set for $A(L / K)$, then so is $a F$ for every $a \in L$ and $\eta(a F)=\eta(F)$ since $k(a F)=L_{a}(k(F))$ and $j(a F)=L_{a}(j(F))$. Thus, for every $a \in A, i(E \cap a H / K)=i\left(a E_{a}\right)$ is a weak Ditkin set for $A(L / K)$ and $\eta(i(E \cap a H / K)) \leq N^{2}$. As $A$ is countable and $\emptyset$ is a Ditkin set for $A(L / K)$, Proposition 1.5 of [8] shows that $E \cap L / K=\bigcup_{a \in A} i\left(a E_{a}\right)$ is a weak Ditkin set for $A(L / K)$ and $\eta(E \cap L / K) \leq N^{2}$. Thus

$$
\left(\left.u\right|_{L}\right)^{N^{2}} \in \overline{\left(\left.u\right|_{L}\right)^{N^{2}} j(E \cap L / K)} .
$$

Since $u=0$ on $G / K \backslash L / K$, it follows that $u^{N^{2}} \in \overline{u^{N^{2}} j(E)}$. Since $u \in k(E)$ was arbitrary, the proof is complete.

For the next proposition compare Proposition 1.6 of [8] and the remark following it.

Proposition 3.3. Let $A$ be a regular and semisimple commutative $B a$ nach algebra satisfying Ditkin's condition at infinity, and let $E$ be a closed subset of $\Delta(A)$. Suppose that each $\varphi \in \Delta(A)$ has a closed relative neighbourhood $E_{\varphi}$ in $E$ which is a weak spectral set and that

$$
\sup \left\{\xi\left(E_{\varphi}\right): \varphi \in E\right\}<\infty .
$$

Then $E$ is a weak spectral set and

$$
\xi(E)=\sup \left\{\xi\left(E_{\varphi}\right): \varphi \in E\right\} .
$$

TheOREM 3.4. Let $G$ be a locally compact group and let $H$ and $K$ be subgroups of $G$ such that $H$ is open, $K$ is compact and $H K$ is a subgroup of $G$. Let $E$ be a closed subset of $G / K$ and for each $a \in G$, let

$$
E_{a}=\{x(H \cap K): x \in H, \text { ax } K \in E\} \subseteq H / H \cap K .
$$

(i) Suppose that $u \in \overline{u A(G / K)}$ for all $u \in A(G / K)$. Then $E$ is a weak spectral set for $A(G / K)$ if and only if every $E_{a}$ is a weak spectral set for $A(H / H \cap K)$ and there is an upper bound for the values $\xi\left(E_{a}\right)$, $a \in G$. More precisely,

$$
\xi(E)=\sup \left\{\xi\left(E_{a}\right): a \in G\right\} .
$$


(ii) Suppose that $G$ is amenable. Then $E$ is a weak Ditkin set for $A(G / K)$ if and only if every $E_{a}$ is a weak Ditkin set for $A(H / H \cap K)$ and there exists an upper bound for the values $\eta\left(E_{a}\right), a \in G$. In this case,

$$
\eta(E) \leq \sup \left\{\eta\left(E_{a}\right)^{2}: a \in G\right\} \leq \eta(E)^{2} .
$$

In particular, $E$ is a Ditkin set for $A(G / K)$ if and only if $E_{a}$ is a Ditkin set for $A(H / H \cap K)$ for all $a \in G$.

Proof. Since $H K$ is an open subgroup of $G$, we can apply Lemma 3.1 with $H K$ in place of $H$. If $E$ is a weak Ditkin set (respectively, weak spectral set) for $A(G / K)$ then, for every $a \in G, a E \cap H K / K$ is a weak Ditkin set (respectively, weak spectral set) for $A(H K / K)$ and $\eta(a E \cap H K / K) \leq \eta(a E)=\eta(E) \quad($ respectively, $\xi(a E \cap H K / K) \leq \xi(E))$. Conversely, suppose that $\eta(a E \cap H K / K) \leq N$ (respectively, $\xi(a E \cap H K / K)$ $\leq N$ ) for all $a \in G$. It then follows from Lemma 3.2 (respectively, Proposition 3.3) and the hypotheses of the theorem that

$$
E=\bigcup_{a \in G}\left(E \cap a^{-1} H K / K\right)=\bigcup_{a \in G} a^{-1}(a E \cap H K / K)
$$

is a weak Ditkin set with $\eta(E) \leq N$ (respectively, weak spectral set with $\xi(E) \leq N)$.

To finish the proof, recall that since $H$ is open and $H K$ is a subgroup of $G$, an application of Lemma 2.3 with $G=H K$ and of the open mapping theorem shows that the restriction map $\left.u \mapsto u\right|_{H}$ is a topological isomorphism from $A(H K / K)$ onto $A(H / H \cap K)$. The corresponding homeomorphism between the Gelfand spaces is simply given by $x(H \cap K) \mapsto x K$, $x \in H$. So $E_{a}$ is a weak Ditkin set for $A(H / H \cap K)$ if and only if $a E \cap H K / K$ is a weak Ditkin set for $A(H K / K)$ and $\eta\left(E_{a}\right)=\eta(a E \cap H K / K)$, and similarly for weak spectral sets.

Combining this with the first part of the proof establishes (i) and (ii).

Corollary 3.5. Let $G, H$ and $K$ be as in Theorem 3.4, and suppose that $H$ has infinite index in $G$. Then weak spectral synthesis holds for $A(G / K)$ if and only if

$$
\sup \{\xi(F): F \subseteq H / H \cap K, F \text { closed }\}<\infty .
$$

Moreover, in this case

$$
\sup \{\xi(E): E \subseteq G / K, E \text { closed }\}=\sup \{\xi(F): F \subseteq H / H \cap K, F \text { closed }\} .
$$

Proof. The "if" part follows immediately from Theorem 3.4(i). For the "only if" part suppose there exists a sequence $\left(F_{n}\right)_{n}$ of closed subsets of $H / H \cap K$ such that $\xi\left(F_{n}\right) \geq n$ for all $n$. Since $G / H$ is infinite, we can choose $a_{n} \in G, n \in \mathbb{N}$, such that $a_{m}^{-1} a_{n} \notin H$ for $n \neq m$. Let

$$
E_{n}=\left\{a_{n} x K: x \in H, x(H \cap K) \in F_{n}\right\} .
$$


Then $E=\bigcup_{n=1}^{\infty} E_{n}$ is a closed subset of $G / K$ and, with the notation of Theorem 3.4, $\left(E_{n}\right)_{a_{n}}=F_{n}$. Therefore,

$$
\xi(E)=\sup \left\{\xi\left(F_{n}\right): n \in \mathbb{N}\right\}=\infty
$$

by Theorem 3.4(i).

4. Towards Malliavin's theorem for weak spectral synthesis in $A(G / K)$. Malliavin's celebrated theorem [13] states that spectral synthesis fails to hold for $A(G)=L^{1}(\widehat{G})$ if $G$ is a nondiscrete locally compact abelian group. By modifying Varopoulos' proof [25] of Malliavin's theorem, it was shown in [18] that the same is true of weak spectral synthesis. Employing this result and a deep theorem due to Zelmanov [27], we proved in [8] that the same conclusion holds for arbitrary nondiscrete locally compact groups $G$. Subsequently, the author was informed by Parthasarathy that this result was also obtained by Prakash and himself.

The problem of when (weak) spectral synthesis holds for $A(G / K)$ appears to be very difficult. It is tempting to conjecture that weak spectral synthesis fails for $A(G / K)$ whenever $K$ is not open in $G$. However, at present we are only able to provide a major step towards confirming this conjecture (Theorem 4.3). We start by pointing out that conversely openness of $K$ is sufficient for weak spectral synthesis to hold for $A(G / K)$.

Lemma 4.1. Let $K$ be a compact open subgroup of $G$. Then

(i) Weak spectral synthesis holds for $A(G / K)$. More precisely, $\xi(E) \leq 2$ for every subset $E$ of $G / K$.

(ii) If $u \in \overline{u A(G / K)}$ for each $u \in A(G / K)$, then spectral synthesis holds for $A(G / K)$.

Proof. Since $A(G / K)$ is Tauberian, (i) follows from [8, Lemma 4.1]. For (ii), note that by hypothesis

$$
u \in \overline{u\left(A(G / K) \cap C_{c}(G / K)\right)}=\overline{u j(\emptyset)}
$$

for every $u \in A(G / K)$. On the other hand, for any subset $E$ of $G / K$, $k(E) j(\emptyset) \subseteq \overline{j(E)}$ and hence

$$
k(E) \subseteq \overline{k(E) j(\emptyset)} \subseteq \overline{j(E)}
$$

(compare the proof of Lemma 4.1 of [8]).

Thus, if $K$ is open in $G$ and $A(G / K)$ has an approximate identity in the weakest possible sense, then spectral synthesis holds for $G$. In particular, if $K$ is open in $G$ and $G$ is amenable, then $A(G / K)$ has a bounded approximate identity (Theorem 1.1) and hence spectral synthesis holds for $A(G / K)$.

The following lemma will be needed to prove the main result of this section. 
LEMMA 4.2. Let $G$ be a compact nilpotent group and $K$ a closed subgroup of $G$. If weak spectral synthesis holds for $A(G / K)$, then $K$ has finite index in $G$.

Proof. Let $Z_{0}=\{e\} \subseteq Z_{1} \subseteq \cdots \subseteq Z_{n}=G$ denote the ascending central series of $G$. We show by induction on $j$ that $K \cap Z_{j}$ has finite index in $Z_{j}$. So let $j \geq 1$, assume that $\left[Z_{j-1}:\left(K \cap Z_{j-1}\right)\right]<\infty$ and consider the chain of subgroups $Z_{j} \supseteq Z_{j} \cap Z_{j-1} K \supseteq Z_{j} \cap K$. Since $Z_{j-1} K / K=Z_{j-1} / K \cap Z_{j-1}$ is finite, we get

$$
\left[\left(Z_{j-1} K \cap Z_{j}\right):\left(K \cap Z_{j}\right)\right] \leq\left[Z_{j-1} K: K\right]<\infty .
$$

Thus it remains to observe that $Z_{j-1} K \cap Z_{j}$ has finite index in $Z_{j}$.

Since weak synthesis holds for $A(G / K)$, it also holds for $A\left(Z_{j} K / K\right)$ and hence for $A\left(Z_{j} K / Z_{j-1} K\right)$. Now, since $Z_{j} / Z_{j-1}$ is contained in the centre of $G / Z_{j-1}$, we see that $Z_{j} K$ normalizes $Z_{j-1} K$. Therefore weak synthesis holds for $A\left(Z_{j} / Z_{j} \cap Z_{j-1} K\right)$. As $Z_{j} / Z_{j-1}$ is abelian, this implies that $Z_{j} / Z_{j} \cap$ $Z_{j-1} K$ is finite, as required.

In what follows, $G_{0}$ always denotes the connected component of the identity of a locally compact group $G$.

TheOREM 4.3. Suppose that $G$ contains a nilpotent open subgroup. If $K$ is a compact subgroup of $G$ and weak synthesis holds for $A(G / K)$, then $K$ is open in $G$.

Proof. Let $H$ be a nilpotent open subgroup of $G$. Since $G / G_{0}$, being totally disconnected, has a neighbourhood basis of the identity consisting of compact open subgroups, we can assume that $H / G_{0}$ is compact. It suffices to show that $H \cap K$ is open in $H$. Since $H$ is open in $G$, weak synthesis holds for $A(H / H \cap K)$ (Theorem 2.2). Thus we can replace $G$ by $H$ and henceforth assume that $G$ is nilpotent and $G / G_{0}$ is compact.

Since $G$ is almost connected, $G$ is a projective limit of Lie groups [14], say $G=\lim _{\alpha} G / K_{\alpha}$. Then weak synthesis holds for each $A\left(\left(G / K_{\alpha}\right) /\left(K_{\alpha} K / K_{\alpha}\right)\right)$ (Lemma 1.4), and since $\left(G / K_{\alpha}\right)_{0}$ has finite index in $G / K_{\alpha}$, weak synthesis also holds for $A\left(\left(G / K_{\alpha}\right)_{0} /\left(K_{\alpha} K / K_{\alpha}\right) \cap\left(G / K_{\alpha}\right)_{0}\right)$. Suppose that we have shown that this implies $\left(G / K_{\alpha}\right)_{0} \subseteq K_{\alpha} K / K_{\alpha}$. Since $G_{0} K_{\alpha} / K_{\alpha} \subseteq$ $\left(G / K_{\alpha}\right)_{0}$, a straightforward argument, using the facts that $K$ is compact and $\bigcap_{\alpha} K_{\alpha}=\{e\}$, shows that $G_{0} \subseteq K$. Then weak synthesis holds for $A\left(\left(G / G_{0}\right) /\left(K / G_{0}\right)\right)$. Since $G / G_{0}$ is a compact nilpotent group, $K$ has finite index in $G$ by Lemma 4.2. This completes the proof.

It therefore remains to verify that if $G$ is a connected nilpotent Lie group and $K$ is a compact subgroup of $G$ such that $A(G / K)$ satisfies weak synthesis, then $K=G$. To that end, let again $Z_{0}=\{e\} \subseteq Z_{1} \subseteq \cdots \subseteq$ $Z_{n}=G$ denote the ascending central series of $G$. We show by induction that $Z_{j} \subseteq K$. Assume that $Z_{j-1} \subseteq K$ for some $j \geq 1$. Then $K$ is normal 
in $Z_{j} K$, since $Z_{j} / Z_{j-1}$ is contained in the centre of $G / Z_{j-1}$ and $Z_{j-1} \subseteq K$. Because weak synthesis holds for $A\left(Z_{j} K / K\right)$, we see that $K$ must be open in $Z_{j} K$. However, $Z_{j} / Z_{j-1}$ is connected and hence $Z_{j} \subseteq K$. This completes the induction step and so $G=Z_{n}=K$.

COROLlarY 4.4. Let $G$ be a locally compact group such that $G_{0}$ is nilpotent and let $K$ be a compact subgroup of $G$. If weak spectral synthesis holds for $A(G / K)$, then $G_{0}$ is contained in $K$.

Proof. Since $G / G_{0}$ is totally disconnected, we can choose an open subgroup $H$ of $G$ such that $H / G_{0}$ is compact. Then weak spectral synthesis holds for $A(H / H \cap K)$. Thus, since $G_{0}=H_{0}$, we can assume that $G=H$. Then $G$, being almost connected, is a projective limit of Lie groups, $G / C_{\alpha}$ say, and as in the proof of Theorem 4.3, it suffices to show that for each $\alpha,\left(G_{0} C_{\alpha}\right) / C_{\alpha}=\left(G / C_{\alpha}\right)_{0}$ is contained in $\left(K C_{\alpha}\right) / C_{\alpha}$. However, this follows from Theorem 4.3 since $\left(G / C_{\alpha}\right)_{0}$ is nilpotent, connected and open in $G / C_{\alpha}$.

We close this section by adding that if $G$ is compact, then a stronger conclusion than the one of Corollary 4.4 can be drawn. Recall that if $H$ is a connected locally compact group, then $H$ possesses a maximal connected normal subgroup, the so-called radical $\operatorname{rad}(H)$ of $H$.

Proposition 4.5. Let $G$ be a compact group and $K$ a closed subgroup of $G$. If weak spectral synthesis holds for $A(G / K)$, then the radical of $G_{0}$ is contained in $K$.

Proof. The usual arguments, already employed in the proofs of Theorem 4.3 and Corollary 4.4, show that we can assume that $G$ is a Lie group. Let $N=\operatorname{rad}\left(G_{0}\right)$. Then $N$ is abelian and hence isomorphic to $\mathbb{T}^{d}$ for some $d \in \mathbb{N}_{0}$. Then there exist $d$ characters $\chi_{1}, \ldots, \chi_{d}$ of $N$ which together separate the points of $N$. For each $j=1, \ldots, d$, the $K$-orbit $K\left(\chi_{j}\right)$ of $\chi_{j}$ in $\widehat{N}$ is finite since $K$ is compact and $\widehat{N}$ is discrete. Thus there exists a subgroup $L$ of $K$ of finite index such that $\chi_{j}\left(k^{-1} x k\right)=\chi_{j}(x)$ for all $x \in N, k \in L$ and $j=1, \ldots, d$. This implies that $L$ centralizes $N$.

Let $H=N L$, which is a subgroup of finite index in $N K$. Then, by Theorem 2.2, weak spectral synthesis holds for $A(N K / K)$ and hence also for the algebra $A(H / H \cap K)$. As $L$ centralizes $N$, it is easy to verify that $H \cap K$ is normal in $H$. Now $H / H \cap K$, being a homomorphic image of $N$, is a connected abelian group. Since weak spectral synthesis fails for any nondiscrete locally compact abelian group [18], it follows that $N \subseteq H$ $\subseteq K$.

5. $A(G / K)$ for certain semidirect products. Since the most useful description of functions $u \in A(G)$ is in terms of $L^{2}$-functions, namely 
$u=f * \check{g}$, where $f, g \in L^{2}(G)$, it is desirable to identify among those convolution products the functions belonging to $A(G / K)$ and to determine the restriction algebra $\left.A(G / K)\right|_{H}$. In concluding the paper we treat this problem in a special semidirect product situation, hoping that this might prove useful in further investigation of groups such as motion groups.

Lemma 5.1. Let $K$ be a compact subgroup of $G$ and let $u \in A(G)$. Then $u \in A(G / K)$ if and only if $u$ is of the form $u=f * \check{g}$, where $f, g \in L^{2}(G)$ and $L_{a} g=g$ for all $a \in K$. Moreover, in this case $f$ and $g$ can be chosen such that $\|u\|=\|f\|_{2}\|g\|_{2}$.

Proof. Normalize Haar measure of $K$ so that $|K|=1$. As pointed out in [3], $G / K$ has a $G$-invariant measure $\mu$, and Weil's formula

$$
\int_{G} f(x) d x=\int_{G / K} \int_{K} f(x k) d k d \mu(x K)
$$

holds. Now, suppose that $u$ is of the indicated form. Then for $x \in G$ and $a \in K$ we have

$$
\begin{aligned}
u(x a) & =\int_{G / K} \int_{K} f(y k) \check{g}\left((y k)^{-1} x a\right) d k d \mu(y K) \\
& =\int_{G / K} \int_{K} f(y k) g\left(a^{-1} x^{-1} y k\right) d k d \mu(y K) \\
& =\int_{G / K} \int_{K} f(y k) g\left(x^{-1} y k\right) d k d \mu(y K)=(f * \check{g})(x)=u(x),
\end{aligned}
$$

whence $u \in A(G / K)$. Conversely, let $u \in A(G / K)$ and let $f, h \in L^{2}(G)$ be such that $u=f * \check{h}$ and $\|u\|=\|f\|_{2}\|h\|_{2}$. Define $g \in L^{2}(G)$ by $g(t)=$ $\int_{K} h(k t) d k$. Then $L_{k} g=g$ for all $k \in K$ and, for any $x \in G$ and $a \in K$,

$$
\begin{aligned}
u(x a) & =\int_{K} u(x k) d k=\int_{G / K} \int_{K} \int_{K} f\left(y k^{\prime}\right) h\left(k^{-1} x^{-1} y k^{\prime}\right) d k d k^{\prime} d \mu(y K) \\
& =\int_{G / K} \int_{K} f\left(y k^{\prime}\right) g\left(x^{-1} y k^{\prime}\right) d k^{\prime} d \mu(y K) \\
& =\int_{G / K} \int_{K} f\left(y k^{\prime}\right) g\left(a^{-1} x^{-1} y k^{\prime}\right) d k^{\prime} d \mu(y K)=(f * \check{g})(x a) .
\end{aligned}
$$

So $u$ has the required form.

Proposition 5.2. Let $G$ be a semidirect product $G=N \rtimes K$, where $K$ is an abelian compact group with dual group $\Gamma$. Let $u \in A(G / K)$. Then there exist $f_{\gamma}, g_{\gamma} \in L^{2}(N), \gamma \in \Gamma$, with the following properties:

(1) $\sum_{\gamma \in \Gamma}\left\|f_{\gamma}\right\|_{2}^{2}<\infty$ and $\sum_{\gamma \in \Gamma}\left\|g_{\gamma}\right\|_{2}^{2}<\infty$. 
(2) For each $\gamma \in \Gamma, g_{\gamma}\left(a^{-1} x a\right)=\gamma(a) g_{\gamma}(x)$ for almost all $x \in N$ and $a \in K$.

(3) $u=f * \check{g}$, where $f, g \in L^{2}(G)$ are defined by

$$
f(x a)=\sum_{\gamma \in \Gamma} f_{\gamma}(x) \overline{\gamma(a)} \quad \text { and } \quad g(x a)=\sum_{\gamma \in \Gamma} g_{\gamma}(x) \gamma(a),
$$

for $x \in N$ and $a \in K$. In this case, the series $\sum_{\gamma \in \Gamma}\left(f_{\gamma} * \check{g}_{\gamma}\right)$ converges in $A(N)$ and equals $\left.u\right|_{N}$.

Conversely, let $v \in A(N)$ and suppose that $f_{\gamma}, g_{\gamma}, \gamma \in \Gamma$, are functions in $L^{2}(N)$ satisfying (1) and (2) and $v=\sum_{\gamma \in \Gamma} f_{\gamma} * \check{g}_{\gamma}$. If $f, g \in L^{2}(G)$ are defined as in (3), then $u=f * \check{g} \in A(G / K)$ and $\left.u\right|_{N}=v$.

Proof. Suppose first that $u \in A(G / K)$. Then, by Lemma 5.1, there exist $f, g \in L^{2}(G)$ such that $u=f * \check{g}$ and $L_{a} g=g$ for all $a \in K$. Since Haar measure of $G$ is the product of Haar measures of $N$ and of $K$, we see that $L^{2}(G)$ is isometrically isomorphic to the Hilbert space tensor product of $L^{2}(N)$ and $L^{2}(K)$. We can assume that Haar measure of $K$ is normalized. Then, since $K$ is compact and abelian, the characters of $K$ form an orthonormal basis of $L^{2}(K)$. Thus $f$ and $g$ admit unique representations

$$
f=\sum_{\gamma \in \Gamma} f_{\gamma} \otimes \bar{\gamma} \quad \text { and } \quad g=\sum_{\gamma \in \Gamma} g_{\gamma} \otimes \gamma,
$$

where $f_{\gamma}, g_{\gamma} \in L^{2}(N)$ and $\sum_{\gamma \in \Gamma}\left\|f_{\gamma}\right\|_{2}^{2}<\infty$ and $\sum_{\gamma \in \Gamma}\left\|g_{\gamma}\right\|_{2}^{2}<\infty$. Explicitly,

$$
f(x a)=\sum_{\gamma \in \Gamma} f_{\gamma}(x) \overline{\gamma(a)} \text { and } g(x a)=\sum_{\gamma \in \Gamma} g_{\gamma}(x) \gamma(a)
$$

for almost all $x \in N$ and $a \in K$. Since $L_{b} g=g$ for each $b \in K$, we also have

$$
g(x a)=g\left(b^{-1} x a\right)=g\left(\left(b^{-1} x b\right) b^{-1} a\right)=\sum_{\gamma \in \Gamma} g_{\gamma}\left(b^{-1} x b\right) \gamma\left(b^{-1} a\right),
$$

which by the uniqueness of the representation of $g$ implies that

$$
g_{\gamma}\left(b^{-1} x b\right)=g_{\gamma}(x) \gamma(b)
$$

for almost all $x \in N$. Using the orthogonality relations for characters, this in turn yields

$$
\begin{aligned}
u(x) & =(f * \check{g})(x)=\int_{N} \int_{K} f(x y a) g(y a) d a d y \\
& =\sum_{\gamma \in \Gamma} \sum_{\delta \in \Gamma} \int_{N} \int_{K} f_{\gamma}(x y) \overline{\gamma(a)} g_{\delta}(y) \delta(a) d a d y \\
& =\sum_{\gamma \in \Gamma} \int_{N} f_{\gamma}(x y) g_{\gamma}(y) d y=\sum_{\gamma \in \Gamma}\left(f_{\gamma} * \check{g_{\gamma}}\right)(x)
\end{aligned}
$$


for almost all $x \in N$. Since

$$
\sum_{\gamma \in \Gamma}\left\|f_{\gamma} * \check{g_{\gamma}}\right\|_{A(N)} \leq \sum_{\gamma \in \Gamma}\left\|f_{\gamma}\right\|_{2}\left\|g_{\gamma}\right\|_{2} \leq\left(\sum_{\gamma \in \Gamma}\left\|f_{\gamma}\right\|_{2}^{2}\right)^{1 / 2}\left(\sum_{\gamma \in \Gamma}\left\|g_{\gamma}\right\|_{2}^{2}\right)^{1 / 2}<\infty,
$$

the series $\sum_{\gamma \in \Gamma}\left(f_{\gamma} * \check{g_{\gamma}}\right)$ converges in $A(N)$ and the limit equals $\left.u\right|_{N}$.

Conversely, let $f_{\gamma}, g_{\gamma} \in L^{2}(N), \gamma \in \Gamma$, be given satisfying (1), (2) and $v=\sum_{\gamma \in \Gamma} f_{\gamma} * \check{g}_{\gamma}$. Then, if $f, g \in L^{2}(G)$ are defined as in (3), we have as above

$$
\begin{aligned}
u(b x) & =\int_{K} \int_{N} f(x y b a) g\left(b^{-1} y b a\right) d y d a \\
& =\sum_{\gamma \in \Gamma} \sum_{\delta \in \Gamma} \int_{N} \int_{K} f_{\gamma}(x y) \overline{\gamma(b a)} g_{\delta}\left(b^{-1} y b\right) \delta(a) d a d y \\
& =\sum_{\gamma \in \Gamma} \int_{N} f_{\gamma}(x y) g_{\gamma}\left(b^{-1} y b\right) \overline{\gamma(b)} d y \\
& =\sum_{\gamma \in \Gamma}\left(f_{\gamma} * \check{g_{\gamma}}\right)(x)
\end{aligned}
$$

for all $x \in N$ and $b \in K$, whence $u \in A(G / K)$ and $\left.u\right|_{N}=v$.

Acknowledgments. The author is grateful to the referee for carefully reading the paper and detecting a number of typos and some inconsistent notation. In addition, Lemma 5.1 was originally only formulated for semidirect products $G=N \rtimes K$. That it holds more generally was pointed out by the referee.

\section{References}

[1] A. Derighetti, Quelques observations concernant les ensembles de Ditkin d'un groupe localement compact, Monatsh. Math. 101 (1986), 95-113.

[2] P. Eymard, L'algèbre de Fourier d'un groupe localement compact, Bull. Soc. Math. France 92 (1964), 181-236.

[3] B. E. Forrest, Fourier analysis on coset spaces, Rocky Mountain J. Math. 28 (1998), 173-190.

[4] B. E. Forrest and N. Spronk, Best bounds for approximate identities in ideals of the Fourier algebra vanishing on subgroups, Proc. Amer. Math. Soc. 134 (2006), 111-116.

[5] B. E. Forrest, E. Samei and N. Spronk, Convolutions on compact groups and Fourier algebras of coset spaces, Studia Math. 196 (2010), 223-249.

[6] C. Herz, Harmonic synthesis for subgroups, Ann. Inst. Fourier (Grenoble) 23 (1973), 91-123.

[7] E. Kaniuth, Weak spectral synthesis for the projective tensor product of commutative Banach algebras, Proc. Amer. Math. Soc. 132 (2004), 2959-2967.

[8] —, Weak spectral synthesis in commutative Banach algebras, J. Funct. Anal. 254 (2008), 987-1002. 
[9] E. Kaniuth, A Course in Commutative Banach Algebras, Grad. Texts in Math. 246, Springer, New York, 2009.

[10] E. Kaniuth and A. T. Lau, A separation property of positive definite functions on locally compact groups and applications to Fourier algebras, J. Funct. Anal. 175 (2000), 89-110.

[11] -, - Spectral synthesis for $A(G)$ and subspaces of $V N(G)$, Proc. Amer. Math. Soc. 129 (2001), 3253-3263.

[12] J. Ludwig and L. Turowska, Growth and smooth spectral synthesis in the Fourier algebras of Lie groups, Studia Math. 176 (2006), 139158.

[13] P. Malliavin, Impossibilité de la synthèse spectrale sur les groupes abéliens non compacts, Inst. Hautes Études Sci. Publ. Math. 2 (1959), 61-68.

[14] D. Montgomery and L. Zippin, Topological Transformation Groups, Wiley, New York, 1966.

[15] T. K. Muraleedharan and K. Parthasarathy, Difference spectrum and spectral synthesis, Tohoku Math. J. 51 (1999), 65-73.

[16] B. D. Park and E. Samei, Smooth and weak synthesis of the anti-diagonal of Lie groups, J. Lie Theory 19 (2009), 275-290.

[17] K. Parthasarathy and R. Prakash, Spectral synthesis in the Fourier algebra and the Varopoulos algebra, Tohoku Math. J. 59 (2007), 441-454.

[18] K. Parthasarathy and S. Varma, On weak spectral synthesis, Bull. Austral. Math. Soc. 43 (1991), 279-282.

[19] J.-P. Pier, Amenable Locally Compact Groups, Wiley, New York, 1984.

[20] H. Reiter and J. D. Stegeman, Classical Harmonic Analysis and Locally Compact Groups, Oxford Univ. Press, Oxford, 2000.

[21] W. Rudin, Fourier Analysis on Groups, Interscience, New York, 1962.

[22] M. Takesaki and N. Tatsuuma, Duality and subgroups, Ann. of Math. 93 (1971), 344-364.

[23] —, -, Duality and subgroups. II, J. Funct. Anal. 11 (1972), 184-190.

[24] N. Th. Varopoulos, Spectral synthesis on spheres, Proc. Cambridge Philos. Soc. 62 (1966), 379-387.

[25] -, Tensor products and harmonic analysis, Acta Math. 119 (1968), 51-112.

[26] C. R. Warner, Weak spectral synthesis, Proc. Amer. Math. Soc. 99 (1987), 244-248.

[27] E. I. Zelmanov, On periodic compact groups, Israel J. Math. 77 (1992), 83-95.

Eberhard Kaniuth

Institut für Mathematik

Universität Paderborn

D-33095 Paderborn, Germany

E-mail: kaniuth@math.uni-paderborn.de

Received February 12, 2009

Revised version November 14, 2009 